\title{
Über das Verhalten der Nucleinbasen bei Verdunkelung von Pflanzen.
}

\author{
Von \\ Alexander Kiesel.
}

(Aus dem pflanzenphysiologischen Laboratorium der Universität Moskau.)

(Der Redaktion zugegangen am 14. Juni 1910.)

Die Nucleinbasen sind als normale Stoffwechselprodukte in höheren Pflanzen schon öfters angegeben worden, ${ }^{1}$ ) ohne daß bei denselben ein näherer Zusammenhang zwischen den einzelnen Vertretern dieser Gruppe nachgewiesen wurde, wie es bereits im Stoffwechsel der Tiere der Fall ist. ${ }^{2}$ )

So wurde hier, nachdem Horbaczewski die Bildung von Harnsäure aus Nucleinbasen nachwies, ${ }^{3}$ ) dieser Prozeß in seine einzelnen Phasen hauptsächlich durch Schitt enhelm und Jones samt Mitarbeitern ${ }^{4}$ ) zerlegt, woraus zu folgern war, daß die genannten Substanzen im folgenden Verhältnis zu einander stehen:

Adenin $+\mathrm{H}_{2} \mathrm{O} \rightarrow$ Hypoxanthin $+\mathrm{O} \rightarrow$ Xanthin $+\mathrm{O} \rightarrow$ Harnsäure Adenase $^{6}$ ) hypoth. Hypoxanthinoxydase Xanthinoxydase $^{6}$ )

$$
\text { Guanin }+\underset{\text { Guanase. }{ }^{7} \text { ) }}{\mathrm{H}_{2} \mathrm{O}} \rightarrow \text { Xanthin }+\mathrm{O} \rightarrow \text { Harnsäure }
$$

Mit der Harnsäurebildung ist der Prozeß aber nicht abgeschlossen, denn die Harnsäure wird ebenfalls zerstört durch Fermente, die Schittenhelm uricolytische Fermente oder $200,250$.

1) Czapek, Biochemie der Pflanzen, Bd. II, S. 70, 179, 194, 199,

2) Sammelreferat von B. Bloch, Bioch. Zentralbl., Bd. V (1906), S. $521,561,817,873$.

3) Monatsh. f. Chemie, Bd. X, S. 624 (1889); Bd. XII, S. 221 (1891).

$\left.{ }^{4}\right)$ Literatur s. Abderhalden, Lehrbuch der physiolog. Chemie, II. Aufl., S. 393.

5) Jones und Winternitz, Diese Zeitschrift, Bd. XLIV, S. 1 (1905).

6) Burian, Diese Zeitschrift, Bd. XLIII, S. 497 (1905).

") Jones und Partridge, Diese Zeitschrift, Bd. XLII, S. 343 (1904). 
Uricooxydasen ${ }^{1}$ ) nennt. Welche Produkte dabei entstehen, darüber ist man noch nicht ganz einig geworden.

So nahm Wiener ${ }^{2}$ ) die Bildung von Glykokoll an. Anderseits wird die Oxalsäurebildung im Organismus oft der Zerstörung der Harnsäure zugeschrieben und der Harnstoff wird ziemlich allgemein als deren Endprodukt angesehen. Endlich hat $\mathrm{W}$ iechowski durch sichere Versuche ${ }^{3}$ ) nachgewiesen, daß Harnsäure durch tierische Gewebe in Allantoin übergeführt wird, welches er für ein nicht weiter im Organismus der Tiere zerfallendes Endprodukt des Nucleinstoffwechsels ansieht. ${ }^{4}$ )

Dieser Befund ist für die Erforschung des Stoffwechsels der Pflanzen von allergrößtem Interesse, da ja bis jetzt in Pflanzen noch niemals Harnsäure und nur für ein pflanzliches Objekt ${ }^{5}$ ) Harnstoff, aber schon öfters Allantoin (bis auf $1 \%$ der Trockensubstanz) aufgefunden wurde. ${ }^{6}$ )

Schon vor 30 Jahren verwies E. Schulze auf die mögliche Verwandtschaft des Allantoins mit dem Hypoxanthin und Xanthin. ?)

Der eben angeführte Vorgang der Veränderung der Nucleinbasen wurde gewissermaßen in Kossels Laboratorium für niedere Pflanzen aufgefunden, indem Baginski das Verschwinden des Xanthins ${ }^{8}$ ) und Schindler einige Jahre später ${ }^{9}$ ) die Entstehung des Hypoxanthins aus Adenin und des Xanthins aus Guanin bei Fäulnis nachwiesen. Außerdem konnte Schindler die allmähliche Veränderung der Nucleinbasen bei Selbstgärung

1) Diese Zeitschrift, Bd. XLV (1905), S. 121, 161; Bd. LXVI (1910), S. 53.

2) Archiv f. exper. Pathol. u. Pharm., Bd. XL, S. 313 (1897); Bd. XLIII, S. 375 (1899).

3) Hofmeisters Beiträge, Bd. IX (1907), S. 295.

4) Biochem. Zeitschrift, Bd. XXV, H. 6, S. 431 (1910).

5) Lycoperdonarten, Bamberger und Landsiedl, Monatshefte f. Chem., Bd. XXIV, S. 218 (1903).

6) Schulze und Barbieri, Journ. f. prakt. Chem., N. F., Bd. XXI, S. 145 (1882); Ber. d. Deutsch. chem. Ges., Bd. XIII, S. 1602 (1881); Schulze und Bosshard, Diese Zeitschrift, Bd. IX, S. 420 (1885).

7) Diese Zeitschrift, Bd. IX, S. 429.

8) Diese Zeitschrift, Bd. VIII, S. 398 (1884).

9) Diese Zeitschrift, Bd. XIII, S. 432 (1889). 
der Hefe nachweisen, wie aus den von ihm für je $400 \mathrm{~g}$ Preßhefe angegebenen Zahlen zu ersehen ist.

\begin{tabular}{l|l|l|l|l|}
\hline & $\begin{array}{c}\text { Frische Hefe } \\
\text { in g }\end{array}$ & $\begin{array}{c}\text { 24 stündige } \\
\text { Selbstgärung in } \mathrm{g}\end{array}$ \\
\hline Adenin . . . . . & 0,1735 & 0,0346 & Spuren & 0 \\
Hypoxanthin . . & 0,3741 & 0,2094 & 0,197 & 0 \\
Guanin. . . . . & 0,115 & 0,104 & 0,0711 & 0 \\
Xanthin . . . . & 0,0982 & 0,1009 & 0,0985 & 0
\end{tabular}

Soweit mir bekannt ist, sind in der Literatur keine Angaben über die Veränderung der Nucleinbasen in höheren Pflanzen vorhanden.

Der von mir im Jahre 1905 im Laboratorium von Prof. E. Schulze ausgeführte Versuch, ${ }^{1}$ ) in dem junge dem Felde entnommene, dicht über dem Boden abgeschnittene Pflanzen von Rotklee teilweise sofort, teilweise nach kurzer Verdunkelung (65 Stunden) verarbeitet wurden, ergab, daß die Quantität der Nucleinbasen nach der Verdunkelung geringer war, als bei der Kontrollportion (S. 75).

Daraus war zu schließen, daß die Nucleinbasen eine Veränderung bei der Verdunkelung erlitten hatten. Ich unternahm deshalb eine neue Untersuchung im folgenden Jahre, die jetzt zum Abschluß gekommen ist.

Die Erwartungen fanden darin ihre volle Bestätigung und es war mir möglich, eine Veränderung der Nucleinbasen nachzuweisen, die dem oben angeführten Schema nahe kommt.

Zur Untersuchung wurden in Blüte sich befindende Pflanzen von Weißklee (Trifolium repens L.) genommen, die im Moskauer Botanischen Garten aufgewachsen waren und das Alter von ca. 3 Monaten hatten (Mai-August).

Die der Erde entnommenen Pflanzen wurden sorgfältig von den ihnen anhaftenden Bodenbestandteilen befreit und in zwei Portionen geteilt. Die erste Portion $(9,65 \mathrm{~kg})$ wurde sofort zur Untersuchung genommen, die zweite $(10,92 \mathrm{~kg}) 77$ Stunden

1) Diese Zeitschrift, Bd. XLIX, S. 72 (1906). 
an dunklem Orte stehen gelassen, wobei sich die unteren Teile (Wurzel) in oft erneutem ausgekochtem Wasser befanden.

Lufttrockensubstanz des Ausgangsmaterials - 11,28\%.

Die Verarbeitung der beiden Portionen geschah in der gleichen Weise. Die frischen zerkleinerten Pflanzen wurden mit heißem Wasser zweimal extrahiert und abgepreßt. Die Extrakte wurden mit Bleiessig gefällt, wobei sie vor der völligen Fällung annähernd auf ihr halbes Volumen eingedampft wurden. Der Überschuß des Bleies wurde mit Schwefelsäure entfernt, der Gehalt der Schwefelsäure auf ca. 3\% gebracht und die basischen Substanzen möglichst vollständig mit Phosphorwolframsäure ausgefällt. Nach dem Zerlegen der Fällung mit Baryt und Entfernen des überschüssigen Baryts mit Kohlensäure wurden in der mit Salpetersäure neutralisierten Flüssigkeit die Nucleinbasen mit salpetersaurem Silber ausgefällt und mit Wasser ausgewaschen.

Die so erhaltenen Silberverbindungen wurden nach den Angaben von Schindler ${ }^{1}$ ) mit Salpetersäure (spez. Gew. 1,1) behandelt und das in der Lösung nach Erkalten und 24stündigem Stehen gebliebene salpetersaure Silberxanthin mit Ammoniak ausgefällt, mit Schwefelwasserstoff zerlegt und eingedampft, wobei sich das Xanthin ausschied.

Es wurde in einer geringen Menge verdünnter Natronlauge gelöst und durch Essigsäure wieder zum Ausscheiden gebracht, wobei sich in der 2. Portion sehr rasch, in der 1. viel langsamer und in viel geringerer Menge schwach gelbe, kleine Krystallaggregate des Xanthins ausschieden.

Das Gewicht des so erhaltenen Rohxanthins betrug:

$$
\begin{aligned}
& \text { I. Portion } 0,0114 \mathrm{~g} \\
& \text { II. } 0,058 \text { » }
\end{aligned}
$$

Beim Umrechnen auf je $10 \mathrm{~kg}$ des Ausgangsmaterials:

I. Portion $0,012 \mathrm{~g}$
II. $\otimes 0,053$ »

Somit konnte eine Zunahme des Xanthins bei der Verdunkelung nachgewiesen werden.

1) Diese Zeitschrift, Bd. XIIl, S. 432 (1889). 
Die Xanthinpräparate wurden durch folgende Reaktionen identifiziert :

1. Nach Abdampfen mit Salpetersäure gab der gelbe Rückstand mit Natronlauge eine schöne rote Färbung, die beim Erwärmen in Purpurrot überging.

2. Eine mit Natronlauge versetzte Lösung von Natriumhypochlorit gab beim Eintragen einer kleinen Menge des Präparats aus der II. Portion einen olivgrünen Ring, der dann ins Braune überging. Die Reaktion bei der I. Portion war lange nicht so gut - der olivgrüne Ring war hier schlecht bemerkbar.

3. Beim Abdampfen einer salzsauren Lösung der Präparate mit Kaliumchlorat gab das aus der II. Portion erhaltene eine rotbräunliche Färbung, die in einer Ammoniakatmosphäre in die charakteristische rosarote überging. Die Reaktion mit der I. Portion ergab keine deutliche Färbung.

4. Das Xanthinpräparat aus der II. Portion wurde durch die charakteristische Form der Silberverbindung unter dem Mikroskop identifiziert. Nach Versetzen einer ziemlich konzentrierten salpetersauren Lösung des Präparats mit salpetersaurem Silber entstand ein weißer Niederschlag, der sich beim Erwärmen auflöste und nach dem Erkalten wieder in Form von kleinen kugelartigen Massen ausschied; dieselben bestanden aus dünnen gebogenen, haarartigen Nadeln. ${ }^{1}$ )

Aus den angeführten Reaktionen folgt mit Sicherheit, daß im Präparate aus den verdunkelten Pflanzen wirklich Xanthin vorlag, welches sich bei dem Verdunkeln gebildet haben mußte.

Die aus der Salpetersäure beim Erkalten und Stehen ausgeschiedenen Silberverbindungen der anderen Nucleinbasen wurden mit warmem Schwefelammonium zerlegt und das Filtrat zur Trockene eingedampft; der Rückstand wurde mit Ammoniak bei gelindem Erwärmen behandelt, um die Trennung des Guanins vom Adenin und Hypoxanthin zu bewirken, die dabei in Lösung gehen mußten. Der ungelöste Rückstand, welcher das Guanin enthalten mußte, wurde mit dem Guanin vereinigt, welches im Schwefelsilberniederschlage zurückgeblieben war und durch

1) Neubauer und Vogel, Anleitung z. qualitativen und quantitativen Analyse des Harns, 1898.

Hoppe-Seyler's Zeitschrift f. physiol, Chemie. LXVII. 
Erwärmen mit Salzsäure ausgezogen und mit Ammoniak gefällt werden konnte. Die vereinigten guaninhaltigen Fraktionen wurden zur Reinigung mit einer geringen Menge schwacher Natronlauge unter schwachem Erwärmen behandelt und das in Lösung gegangene Guanin mit Alkohol und Essigsäure ausgefällt. Aus der I. Portion schied sich das Guanin allmählich in Form von charakteristischen Drusen aus, dagegen konnte aus der II. Portion nur eine kleine Menge eines flockigen Niederschlages erhalten werden, der keine Guaninreaktionen gab.

Die Menge des Guanins betrug:

I. Portion $0,0601 \mathrm{~g}$, auf $10 \mathrm{~kg}$ berechnet $0,062 \mathrm{~g}$

II. $>0$

Das nach beschriebenem Verfahren erhaltene Guaninpräparat gab folgende Reaktionen:

1. Beim Verdampfen mit Salpetersäure blieb ein gelblicher Rückstand nach, der mit Natronlauge eine rote Färbung gab, die beim Erwärmen in eine blaurote überging.

2. Mit Ferricyankalium gab das Präparat beim Stehen rote prismatische Drusen.

3. Mit Metaphosphorsäure bildete sich sehr bald ein feiner weißer Niederschlag.

4. Mit Pikrinsäure versetzt schied sich das nadelförmige Pikrat aus.

5. Beim Überführen in das salzsaure Salz wurden die für dieses Salz charakteristischen feinen langen Nadeln erhalten.

Demnach mußte Guanin vorliegen, welches sich bei dem Verdunkeln in der II. Portion umgewandelt haben mußte. Es wäre mit Rücksicht auf die für das Xanthin erhaltenen Resultate an eine Bildung von Xanthin aus dem Guanin zu denken.

Die beim Digerieren in Ammoniak gelöste Menge der Nucleinbasen wurde zur langsamen Entfernung des Ammoniaks stehen gelassen, wobei sich ein feiner krystallinischer Niederschlag bildete. Da man aber nicht sicher sein konnte, auf diese Weise eine quantitative Trennung des Adenins vom Hypoxanthin zu bewirken, wurde eine Trennung nach Bruhns ${ }^{1}$ )

1) Diese Zeitschrift, Bd. XIV, S. 533 (1890). 
mit $1,1 \%$ wässeriger Pikrinsäure im wiederaufgelösten Niederschlage, sowie in der Flüssigkeit getrennt vorgenommen.

Bei der Verarbeitung der I. Portion konnte aus dem in Wasser aufgelösten Niederschlage und ebenfalls in der Flüssigkeit eine sofortige Fällung des Adeninpikrates (zusammen $0,47+0,12=0,59 \mathrm{~g}$ ) erhalten werden, welche sogleich abgenutscht und mit kaltem Wasser ausgewaschen wurde.

Die II. Portion gab keine sofortige Fällung mit Pikrinsäure, wie in der Lösung des Niederschlages, so auch in der Flüssigkeit. Bei 2 tägigem Stehen bildeten sich aber in der Lösung des Niederschlages tafelförmige Krystalle eines Pikrates $(0,223 \mathrm{~g})$, die kein Adeninsalz darstellen konnten.

Aus der Menge des Adeninpikrates konnte die Menge des Adenins berechnet werden:

I. Portion $0,59 \mathrm{~g}$ Pikrat $=0,22 \mathrm{~g}$ freies Adenin, auf $10 \mathrm{~kg}$ berechnet: $0,228 \mathrm{~g}$.

II. Portion 0.

Zur Identifikation des Adenins wurde aus dem Pikrat das freie Adenin wiedergewonnen und mit demselben die Reaktionen vorgenommen. Das Verfahren war folgendes:

Das Pikrat wurde mit 10\%iger. Schwefelsäure zerlegt und die Pikrinsäure durch Ausschütteln mit Äther entfernt. Nach Entfernen der Schwefelsäure durch Baryt aus heißer Lösung und des überschüssigen Bary̧ums mit Kohlensäure wurde das Filtrat zur Trockene eingedampft, in heißem Wasser aufgenommen und wieder eingedampft, wobei ein feiner krystallinischer Rückstand erhalten wurde, der dann zu den Reaktionen genommen wurde.

1. Die Xanthinprobe war negativ.

2. Nach $1 / 2$ stündigem Erwärmen im Wasserbade mit Zink und Salzsäure, wobei sich eine vorübergehende deutliche Rosafärbung bemerkbar machte, wurde Natronlauge bis zur starken alkalischen Reaktion zugesetzt und von Zeit zu Zeit geschüttelt. Nach einiger Zeit entstand in der Flüssigkeit die charakteristische Rosafärbung.

3. Nach Auflösen in schwacher Salzsäure (getrennt vorgenommen für die beiden erhaltenen und zerlegten Pikratfäl- 
lungen) von 3-5 mg der Adeninpräparate wurde tropfenweise ein berechneter Überschuß von Goldchloridlösung zugesetzt. Beim Stehen im Exsikkator schieden sich nach wenigen Stunden die charakteristischen orangefarbigen, mit schiefen Flächen versehenen Prismen des Goldchloriddoppelsalzes aus.

4. Mit essigsaurem Kupfer gaben die Präparate keine Fällung, was auf die Abwesenheit von Hypoxanthin in denselben hinweist.

Daraus ist also zu ersehen, daß das Adenin, welches in normalen Pflanzen enthalten war, bei Verdunkelung der Pflanzen bis auf nicht nachweisbare Spuren oder sogar vollständig verschwunden war, was wohl doch eher durch destruktive Prozesse zu erklären ist.

Die beim Stehen aus der II. Portion (s. oben) ausgeschiedenen tafelförmigen Krystalle des Pikrats, welches vermutlich eine Verbindung des Hypoxanthins darstellte, die sich beim Stehen in den genannten Bedingungen ausscheiden konnte, wurden mit 10\% iger Schwefelsäure zerlegt und die Pikrinsäure durch Ausschütteln mit Äther entfernt. Nach dem Entfernen der Schwefelsäure durch Baryt und des Überschusses vom letzteren durch Kohlensäure wurde die Flüssigkeit zur Trockene verdampft, der Rückstand in heißem Wasser gelöst und wieder eingedampft.

Mit einem kleinen Teile des Rückstandes wurden folgende Reaktionen vorgenommen:

1. Die Xanthinreaktion - völlig negativ.

2. Mit essigsaurem Kupfer bildete sich sofort in der Kälte ein hellgrüner flockiger Niederschlag, wie es für das Hypoxanthin charakterisiert ist.

3. Ein kleiner Teil des Rückstandes wurde in verdünnter Salzsäure gelöst und mit Goldchlorid versetzt. Es entstanden beim Stehen im Exsikkator dabei keine für das Adenin charakteristischen Krystalle.

Der größte Teil des Präparates wurde in schwacher Salpetersäure gelöst, ein wenig eingedampft und stehen gelassen, wobei die sehr charakteristischen wetzsteinförmigen Krystalle des Hypoxanthinnitrats erhalten wurden. 
Demnach konnte ein Teil des Hypoxanthins aus der II. Portion in Form des Pikrats erhalten werden.

Die nach dem Ausfällen der Pikrate erhaltenen Lösungen aus beiden Portionen wurden in der schon öfters angegebenen Weise von der Pikrinsäure befreit und eingedampft.

Nach dem Aufnehmen der Rückstände in heißem Wasser wurde das in Lösung gegangeneHypoxanthin mit ammoniakalischer Silbernitratlösung gefällt, ausgewaschen und mit Schwefelwasserstoff zerlegt. Die zur Trockene eingedampften Filtrate wurden in heißem Wasser aufgenommen und wieder abgedampft. Die so erhaltenen Rückstände (freies Hypoxanthin) wurden gewogen:

I. Portion $0,055 \mathrm{~g}$, auf $10 \mathrm{~kg}$ berechnet: $0,057 \mathrm{~g}$.

II. Portion $0,070 \mathrm{~g}$, mit dem aus dem Pikrat $(0,223 \mathrm{~g})$ erhaltenen: $0,070+0,079=0,149 \mathrm{~g}$, auf $10 \mathrm{~kg}$ berechnet $0,136 \mathrm{~g}$.

Mit einem Teil der Präparate wurden die oben unter 1 und 2 für das Hypoxanthin angegebenen Reaktionen ausgeführt mit dem gleichen Ergebnis wie dort.

Der Rest der aus beiden Portionen erhaltenen Präparate wurde in das Nitrat übergeführt, wobei die schon angegebene charakteristische Krystallform des Hypoxanthinnitrats für beide Präparate erhalten wurde.

Daraus ist zu ersehen, daß während der Verdunkelung eine Vergrößerung der Hypoxanthinmenge in den Pflanzen stattgefunden hat, und voraussichtlich hatte sich diese aus dem verschwundenen Adenin gebildet.

Die erhaltenen Resultate lassen sich in folgender Tabelle zusammenstellen:

\begin{tabular}{c|c|c|c}
\hline & $\begin{array}{c}\text { Kontrollportion (I) } \\
\mathbf{g}\end{array}$ & $\begin{array}{c}\text { Verdunkelte Pflanzen } \\
\mathbf{g}\end{array}$ & $\begin{array}{c}\text { Differenz } \\
\mathbf{g}\end{array}$ \\
\hline Guanin . . . . & 0,062 & 0 & $-0,062$ \\
Xanthin . . . . & $0,012($ ?) & 0,053 & $+0,041$ \\
Adenin . . . . & 0,228 & 0 & $-0,228$ \\
Hypoxanthin. . & 0,057 & 0,136 & $+0,079$
\end{tabular}

Die Zahlen drücken die Menge der freien Nucleinbasen aus und beziehen sich auf je $10 \mathrm{~kg}$ des entsprechenden frischen Materials oder auf je $1128 \mathrm{~g}$ Lufttrockensubstanz. 
Wenn man die Menge der Nucleinbasen für jede Portion summarisch ausdrückt, so erhält man:

I. Portion - 0,359 $\mathrm{g}$ freier Nucleinbasen

II. $\gg-0,189 \mathrm{~g}$ »

Somit hatte ich auch die Bestätigung meiner früheren Angabe ${ }^{1}$ ) über die Verminderung der Nucleinbasenmenge bei Verdunkelung von Rotklee in diesem Falle bei Weißklee erhalten. Die Veränderung der Nucleinbasen bestand in einer starken Desamidation, die von weiterem Zerfall begleitet war, die wohl, wie im Tierreich, der Tätigkeit von Fermenten zuzuschreiben sind.

Die Methoden, welche wir für die Isolierung der Nucleinbasen kennen, sind wohl nicht ohne Verlust, dennoch glaube ich, daß bei sorgfältigem gleichartigem Verfahren man zu vergleichbaren Zahlen kommen kann und die Unvollkommenheit der Methoden die erhaltenen Resultate nicht hinfällig macht.

Ein anderer Versuch, vorgenommen mit Rotklee, sollte entscheiden, ob sich nicht etwa bei Verdunkelung doch Harnsäure in Pflanzen bilden sollte.

Die Verdunkelung dauerte 5 Tage lang, wobei für die Kontrollportion, in der auch die Nucleinbasen untersucht wurden, $8,3 \mathrm{~kg}$, für die verdunkelte Versuchsportion $7,2 \mathrm{~kg}$ frischer Pflanzen verwendet wurden.

Das Verfahren zum Ausscheiden der Harnsäure entsprach den Angaben von Krüger und Salomon, ${ }^{2}$ ) wobei zuerst die durch Bleiessig fällbaren Substanzen entfernt und das überschüssige Blei durch Schwefelwasserstoff ausgeschieden wurde.

In beiden Portionen konnte ich nicht die geringste Spur Harnsäure nachweisen.

In der Kontrollportion wurden nach dem Abtrennen der «Harnsäure» die 4 Nucleinbasen Xanthin, Guanin, Adenin und Hypoxanthin nach dem oben für Weißklee beschriebenen Verfahren nachgewiesen.

Die Entscheidung der Frage, ob sich bei dem Verschwinden der Nucleinbasen in Pflanzen Allantoin bildet, soll den Gegenstand einer nächsten Untersuchung bilden.

1) 1. c. 2) Diese Zeitschrift, Bd. XXVI, S. 373. 